\title{
Utilidad de la monitorizacion de la PIC en pacientes con sospecha de disfunción
} valvular

\author{
A. Horcajadas; A. Román; G. Olivares; E. Saura; A. Jorques; N. Cordero; B. Ibáñez; C. Sánchez y M.A. Roldán
}

Hospital Universitario Virgen de las Nieves. Granada.

\section{Resumen}

La sospecha de disfunción valvular constituye una situación frecuente en neurocirugía. En numerosas ocasiones ni la clínica, ni la exploración, ni la radiología pueden confirmar o descartar el diagnóstico. La monitorización continua de la PIC es un método seguro y fiable que aporta valiosa información acerca de la situación de la dinámica del LCR en estos pacientes. El sistema de registro debe permitir no sólo el análisis cuantitativo de la PIC sino también morfológico del trazado, para identificar ondas patológicas (como las B de alta amplitud) relacionadas con situaciones de disfunción valvular. En el presente trabajo se recoge la experiencia de monitorización continua de la PIC en pacientes con sospecha de disfunción valvular sin criterios clínicos y radiológicos concluyentes, en los que el análisis morfológico del registro permitió un diagnóstico fiable que se tradujo en la mejoría de todos los pacientes tratados conforme a este criterio. El método utilizado fue un sensor intraparenquimatoso Camino ${ }^{\circledR}$ con software de recogida y representación neuroPICture $\subseteq$ (desarrollado por el primer autor). Las complicaciones relacionadas con la monitorización fueron nulas y el registro obtenido útil para las objetivos propuestos.

PALABRAS CLAVE. Monitorización PIC. Disfunción valvular. Ondas B. NeuroPICture

Usefulness of IPC continuous monitoring in shunt dysfunction

\section{Summary}

Shunt dysfunction is a common situation in neurosurgery. Often symptoms, physical examination and radiology are not enough to set a diagnosis. ICP continuous monitoring is a safe and reliable tool that provides

Recibido: 25-07-10. Aceptado: 7-11-10 valuable information about CSF dynamics in these patients. Not only quantitative analysis is needed but also a qualitative one that enables pathological waves identification, because high amplitude $B$ waves are strongly related to shunt dysfunction. In this paper experience about ICP continuous monitoring in patients with shunt dysfunction suspect is presented. Quantitative and qualitative data analysis led to a correct diagnosis, improving all the patients treated according to this criterion. An intraparenchymatous Camino® sensor and neuroPICture software (developed by first author) for data collection and graphic representation were used. Complications related to monitoring were absent and graphics obtained useful for qualitative analysis.

KEY WORDS. ICP monitoring. Shunt dysfunction. B waves. NeuroPICture.

\section{Introducción}

Los pacientes portadores de derivaciones de LCR constituyen un grupo importante en la práctica neuroquirúrgica. La prevalencia estimada de hidrocefalia se sitúa en torno al 1-1,5\%, siendo la incidencia de la hidrocefalia congénita de 0,9 a 1,8/1000 nacimientos. Hasta la llegada de las técnicas endoscópicas el tratamiento casi exclusivamente empleado ha sido la derivación de LCR desde el sistema ventricular al atrio inicialmente, y al peritoneo con posterioridad. Las causas más frecuentes de hidrocefalia son las hemorragias intracerebrales (subaracnoidea, intraventricular), las malformaciones (Chiari, estenosis de acueducto de Silvio), las infecciones (meningitis, ventriculitis) y los tumores. La mayoría de los pacientes se convierten en sujetos dependientes de estos sistemas valvulares de manera que la disfunción de los mismos se traduce en clínica de hipertensión intracraneal. Si tenemos en cuenta esta circunstancia, la incidencia de la hidrocefalia y el hecho

Abreviaturas. LCR: líquido cefalorraquídeo. PIC: presión intracraneal. 
de que las válvulas, al tratarse de dispositivos artificiales, tienen una vida media limitada, la frecuencia de problemas derivados de su mal funcionamiento en el quehacer del neurocirujano supone un porcentaje importante de su práctica habitual. Las disfunciones valvulares generalmente debutan con cuadros de hipertensión intracraneal aguda o subaguda, reconocibles tanto desde el punto de vista clínico como radiológico. Sin embargo, en determinadas ocasiones, la sintomatología es más leve y las exploraciones complementarias anodinas o inespecíficas, haciendo que el diagnóstico de hipertensión intracraneal sea más difícil. La presencia de cefalea acompañada o no de náuseas y vómitos, puede darse en sujetos con disfunción valvular pero también en otros pacientes portadores de derivaciones que funcionan correctamente y cuyos síntomas no pueden ser atribuidos a ellas. Asimismo pueden darse cuadros graves de hipertensión intracraneal en contextos clínicos poco llamativos. La radiología tampoco supone una gran ayuda, puesto que suele tratarse o bien de pacientes con ventrículos dismórficos, con derivaciones de largo tiempo de evolución, en los que establecer el diagnóstico radiológico de hidrocefalia no es fácil, o bien de pacientes con ventrículos escasamente elásticos, en los que pequeños cambios de tamaño ventricular que pueden pasar desapercibidos pueden traducir importantes aumentos de la PIC. En el contexto de los pacientes portadores de "shunts" existe además una situación radiológica denominada ventrículos en hendidura caracterizada por un tamaño ventricular menor del normal, en los que la presión intracraneal puede estar elevada, normal o excesivamente disminuida con síntomas similares en las tres situaciones.

La monitorización de la PIC constituye por tanto en este tipo de pacientes una herramienta de gran importancia para establecer el diagnóstico y plantear las alternativas terapéuticas.

\section{Material y métodos}

Desde marzo de 2005 a septiembre de 2009 se estudiaron 33 pacientes portadores de derivaciones de LCR en los que se sospechaba una disfunción valvular por sintomatología clínica compatible (cefalea, alteraciones de la marcha, deterioro cognitivo o trastornos visuales) y diagnóstico radiológico no concluyente. Se recoge la sintomatología y la puntuación en la escala de Karnofski.

La técnica de monitorización se basó en la colocación de un sensor intraparenquimatoso Camino ${ }^{\circledR}$ bajo anestesia local en quirófano y sistema de captura y representación gráfica neuroPICture $\odot$. La monitorización se mantenía por un espacio mínimo de 72 horas, o menor en caso de que el registro fuera lo suficientemente demostrativo antes de ese límite.

Se recogen las cifras de PIC media total, diurna y noc- turna, así como las de PIC sistólica y diastólica. Se evalúa el trazado y se registra la presencia de ondas A plateau de Lundberg (entendidas como elevaciones de ascenso rápido e inicio súbito hasta valores de entre 60 y $80 \mathrm{mmHg}$, manteniéndose a esos niveles durante un tiempo que oscila entre 5 y 20 minutos, para volver a descender a valores normales de forma también brusca ${ }^{15}$ ), ondas A preplateau (con una morfología similar a las "plateau", pero de menor amplitud y duración ${ }^{20}$ ) y ondas B (de menor amplitud y duración que presentan un ascenso paulatino de la PIC, sin meseta, seguida de una caída brusca, y que para ser consideradas como tales han de presentarse agrupadas en trenes de ondas con un frecuencia que oscila entre 0,5 y 2 ondas por minuto ${ }^{15}$ ) que a su vez pueden clasificarse en ondas B de alta amplitud $(>10 \mathrm{mmHg})$ y de baja amplitud $(<10$ $\mathrm{mmHg}$ ). Conforme al análisis morfológico del trazado se evalúa el registro en normal, patológico o dudoso. Se recogen las complicaciones relacionadas con la monitorización. Los resultados se valoran a los seis meses mediante entrevista personal en la que se evalúa la evolución de cada uno de los síntomas que motivaron la monitorización puntuándolos en 1 si hay mejoría, 0 si no ha cambiado y -1 si ha empeorado. Asimismo se recoge la puntuación en la escala de Karnofski en ese momento.

\section{Resultados}

Desde el punto de vista descriptivo, apenas si hubo diferencias en cuanto a sexo (18 hombres y 15 mujeres). Las edades de los pacientes oscilaron entre los 9 y los 71 años, con una edad media de 36.8 años.

Las causas de hidrocefalia se recogen en la tabla 1. La situación más frecuente fue la de pacientes con derivaciones de LCR desde el nacimiento, que constituyeron algo más del $45 \%$ de la serie. Estos pacientes en su mayoría habían recibido la válvula a raíz de hemorragias perinatales o en el contexto de un mielomeningocele. El resto de las causas se reparten de forma más o menos equitativa entre las hidrocefalias de origen tumoral, hemorrágico,

\begin{tabular}{|lr|}
\multicolumn{1}{c}{$\begin{array}{c}\text { Tabla 1 } \\
\text { Causas DVP }\end{array}$} \\
\hline Hidrocefalia connatal & 15 \\
\hline Hidrocefalia tumoral & 4 \\
\hline Hidrocefalia malformativa & 4 \\
\hline Hidrocefalia crónica del adulto & 3 \\
\hline Hidrocefalia posthemorrágica & 3 \\
\hline Hidrocefalia postraumática & 3 \\
\hline Hidrocefalia postinfecciosa & 1 \\
\hline
\end{tabular}


traumático y malformativo (Chiari y estenosis de Silvio), siendo las de naturaleza infecciosa las menos frecuentes, con un solo caso de la serie.

La edad de los dispositivos de derivación de LCR, es decir, el tiempo que llevaban colocadas en el paciente, oscilaba entre 1 mes y 32 años, con una media de 6,3 años. En el grupo de pacientes portadores de válvulas desde el nacimiento la antigüedad de las mismas era considerablemente mayor, siendo la media de 12,25 años, casi cinco veces que en el resto del grupo (2,5 años). En el gráfico se representa la edad media de las derivaciones en cada uno de los grupos (Figura 1).

El síntoma más frecuente fue la cefalea, presente hasta en el $69.7 \%$ de los pacientes. De estos pacientes 4 se quejaban además de un deterioro visual, presentando papiledema tres de ellos. El resto de los pacientes presentaban deterioro cognitivo, alteraciones esfinterianas o trastornos de la marcha, síntomas que no habían mejorado o que habían reaparecido o empeorado tras una derivación de LCR (Figura 2).

La exploración física no aportó datos diagnósticos salvo el papiledema referido anteriormente. La exploración del reservorio valvular tampoco resultó de utilidad para apun-

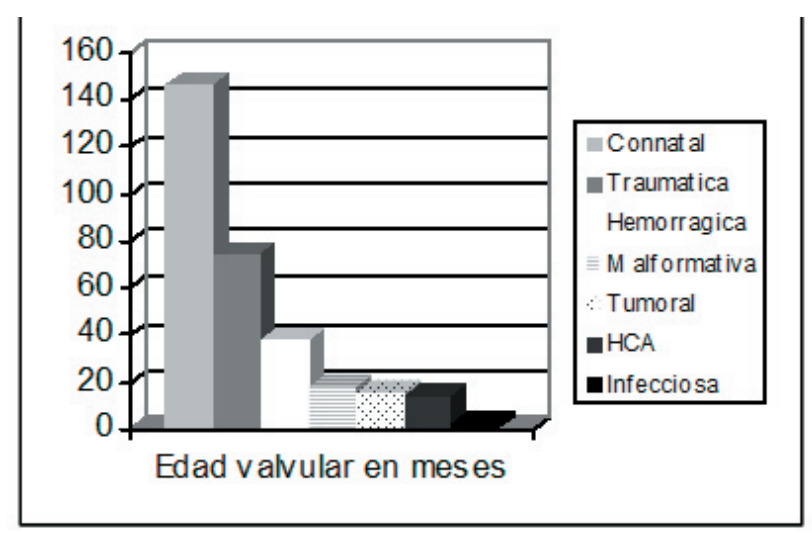

Figura 1

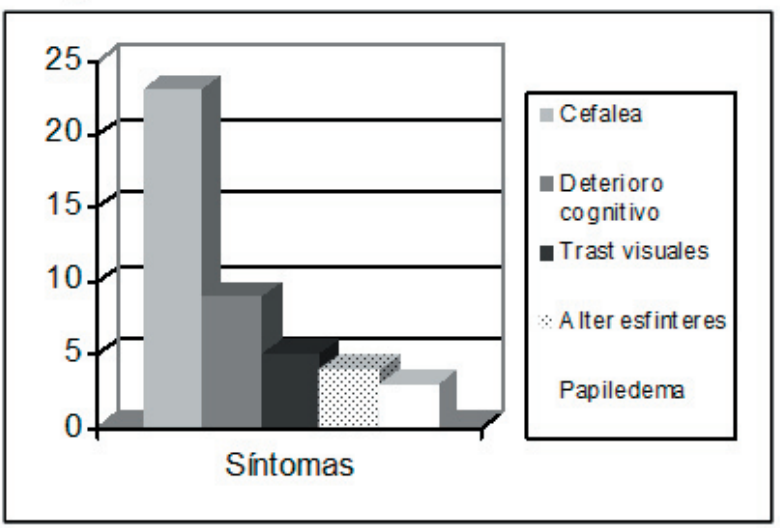

Figura 2

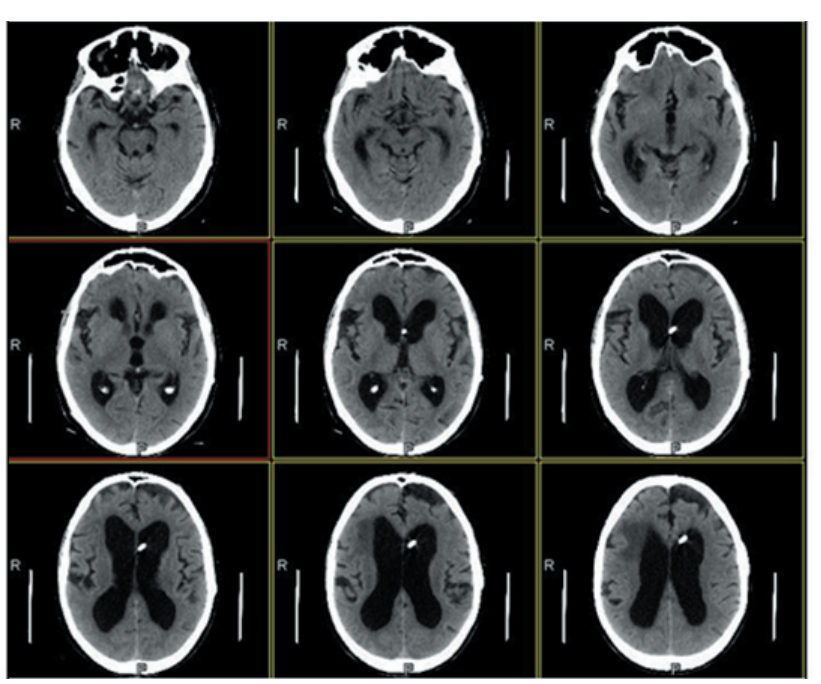

Figura 3. TAC craneal que muestra dilatación ventricular sin signos de actividad con catéter bien colocado.

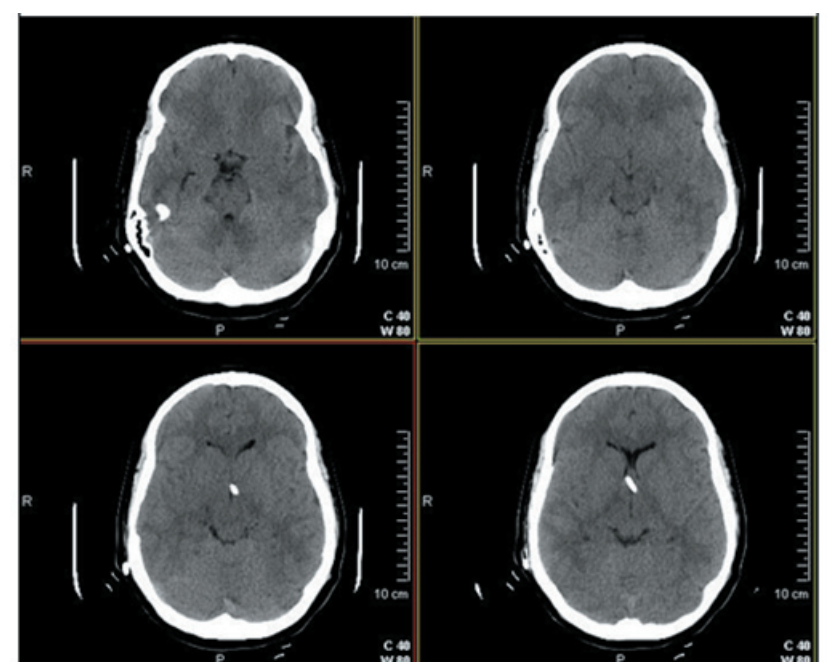

Figura 4. TAC craneal que muestra unos ventrículos en hendidura con catéter ventricular en III ventrículo.

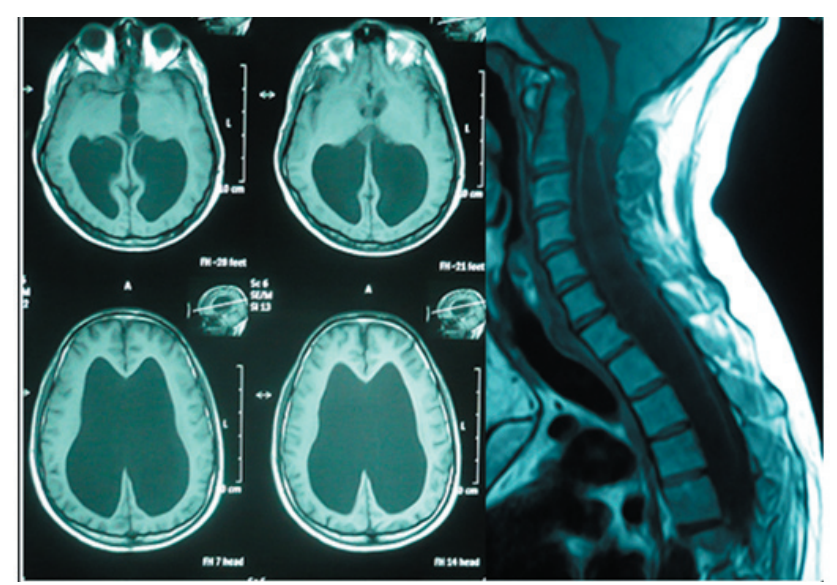

Figura 5. Dilatación ventricular y siringomielia en paciente con DVP connatal en el contexto de mielomeningocele $y$ malformación de Chiari. 
Tabla 2

\begin{tabular}{|c|c|c|c|c|c|c|c|c|c|c|c|c|}
\hline Id & Ed & Sx & Causa DVP & Clinica & $\begin{array}{c}\text { Meses } \\
\text { valv }\end{array}$ & $\begin{array}{c}\text { PIC } \\
\text { media }\end{array}$ & $\begin{array}{l}\text { A } \\
\text { Plat }\end{array}$ & $\begin{array}{c}\text { A } \\
\text { Prepla }\end{array}$ & $\begin{array}{c}\text { B } \\
\text { alta }\end{array}$ & $\begin{array}{c}\text { B } \\
\text { baja }\end{array}$ & $\%$ В & $\begin{array}{l}\text { Valoracion } \\
\text { registro }\end{array}$ \\
\hline 1 & 32 & $\mathrm{H}$ & HCF connatal & Cefalea & 384 & 5,87 & No & No & Sí & Sí & 80 & PATOLOGICO \\
\hline 2 & 12 & M & HCF connatal & $\begin{array}{l}\text { Cefalea, deterioro visual } \\
\text { y vómitos. Papiledema }\end{array}$ & 144 & 59,6 & Sí & Sí & Sí & No & 100 & PATOLOGICO \\
\hline 3 & 66 & $\mathrm{M}$ & HCF malform & $\begin{array}{l}\text { Deterioro cognitivo, } \\
\text { marcha y esfínteres }\end{array}$ & 12 & $-2,47$ & No & No & No & Sí & 20 & NORMAL \\
\hline 4 & 67 & $\mathrm{H}$ & HCA & $\begin{array}{l}\text { Alteración cognitiva } \\
\text { y esfínteres }\end{array}$ & 15 & 7,43 & No & No & No & Sí & 35 & PATOLOGICO \\
\hline 5 & 22 & M & HCF connatal & $\begin{array}{l}\text { Mielomeninocele. } \\
\text { Cefalea episódica }\end{array}$ & 36 & 7,11 & Sí & Sí & Sí & Sí & 80 & PATOLOGICO \\
\hline 6 & 53 & M & HCF tumoral & $\begin{array}{l}\text { Cefalea y deterioro } \\
\text { visual. Papiledema }\end{array}$ & 11 & 9,84 & No & No & No & Sí & 15 & DUDOSO \\
\hline 7 & 65 & $\mathrm{H}$ & HCF postHSA & $\begin{array}{l}\text { Alteración cognitiva } \\
\text { y esfínteres }\end{array}$ & 108 & $-4,41$ & No & No & No & No & & NORMAL \\
\hline 8 & 12 & $\mathrm{H}$ & HCF tumoral & Cefalea & 24 & 8,28 & No & No & Sí & Sí & 15 & DUDOSO \\
\hline 9 & 27 & $\mathrm{H}$ & HCF connatal & Cefalea & 204 & 7,68 & No & No & Sí & Sí & 100 & PATOLOGICO \\
\hline 10 & 13 & $\mathrm{H}$ & HCF connatal & Cefalea y diploplia & 120 & 26,55 & Sí & Sí & Sí & Sí & 100 & PATOLOGICO \\
\hline 11 & 13 & $\mathrm{H}$ & HCF connatal & Cefalea & 132 & 24,28 & Sí & Sí & Sí & Sí & 100 & PATOLOGICO \\
\hline 12 & 43 & M & HCF tumoral & Cefalea, deterioro visual & 11 & 5,99 & No & No & No & Sí & 15 & NORMAL \\
\hline 13 & 23 & M & HCF connatal & Cefalea y vómitos & 244 & 26,13 & Sí & No & Sí & Sí & 100 & PATOLOGICO \\
\hline 14 & 18 & M & HCF connatal & Cefalea & 60 & 17,8 & No & No & Sí & Sí & 100 & PATOLOGICO \\
\hline 15 & 66 & M & HCF postHSA & Deterioro cognitivo & 1 & $-0,41$ & No & No & No & Sí & 35 & DUDOSO \\
\hline 16 & 48 & M & HCF malform & Cefalea, alteración marcha & 5 & 10,23 & No & Sí & Sí & Sí & 100 & PATOLOGICO \\
\hline 17 & 48 & M & HCF malform & Cefalea, alteración marcha & 6 & 8,12 & No & Sí & Sí & Sí & 100 & PATOLOGICO \\
\hline 18 & 16 & M & HCF malform & Cefalea & 48 & 4,93 & No & No & No & No & 0 & NORMAL \\
\hline 19 & 52 & $\mathrm{H}$ & HCF postinfecc & Cefalea & 3 & $-0,34$ & No & No & No & Sí & 80 & PATOLOGICO \\
\hline 20 & 71 & M & HCA & Triada Hakim & 20 & 9,4 & No & No & No & No & & NORMAL \\
\hline 21 & 65 & $\mathrm{H}$ & HCF tumoral & Triada Hakim & 24 & 3,55 & No & No & No & Sí & 15 & NORMAL \\
\hline 22 & 14 & $\mathrm{H}$ & HCF connatal & Cefalea y vómitos & 24 & 16,2 & Sí & Sí & Sí & Sí & 100 & PATOLOGICO \\
\hline 23 & 64 & $\mathrm{H}$ & HCF postraum & Cefalea, deterioro visual & 60 & 2,51 & No & No & No & No & 0 & NORMAL \\
\hline 24 & 24 & $\mathrm{H}$ & HCF connatal & $\begin{array}{l}\text { Cefalea, trastorno marcha. } \\
\text { Siringomielia }\end{array}$ & 288 & 5,07 & No & No & Sí & Sí & 70 & PATOLOGICO \\
\hline 25 & 48 & M & HCF postHSA & Cefalea & 5 & 3,01 & No & No & No & No & 0 & NORMAL \\
\hline 26 & 65 & $\mathrm{H}$ & HCA & Triada Hakim & 8 & 3,27 & No & No & No & No & 0 & NORMAL \\
\hline 27 & 20 & $\mathrm{M}$ & HCF connatal & Cefaleas & 26 & 4,85 & No & No & No & Sí & 10 & NORMAL \\
\hline 28 & 43 & $\mathrm{H}$ & HCF postraum & $\begin{array}{l}\text { Cefaleas. Deterioro } \\
\text { cognitivo }\end{array}$ & 7 & 2,2 & No & No & No & Sí & 80 & PATOLOGICO \\
\hline 29 & 9 & $\mathrm{H}$ & HCF connatal & Cefaleas y vómitos & 108 & 30,15 & No & Sí & Sí & Sí & 10 & PATOLOGICO \\
\hline 30 & 9 & $\mathrm{H}$ & HCF connatal & $\begin{array}{l}\text { Cefaleas y vómitos. } \\
\text { Deterioro visual }\end{array}$ & 120 & 42,62 & Sí & Sí & Sí & Sí & 100 & PATOLOGICO \\
\hline 31 & 38 & $\mathrm{H}$ & HCF postraum & Cefaleas & 156 & 2,87 & No & No & No & No & 0 & NORMAL \\
\hline 32 & 20 & M & HCF connatal & Cefaleas & 48 & 6,08 & No & No & No & No & 0 & NORMAL \\
\hline 33 & 29 & $\mathrm{H}$ & HCF connatal & Cefaleas y diploplia & 60 & 43,58 & Sí & No & Sí & No & 100 & PATOLOGICO \\
\hline
\end{tabular}




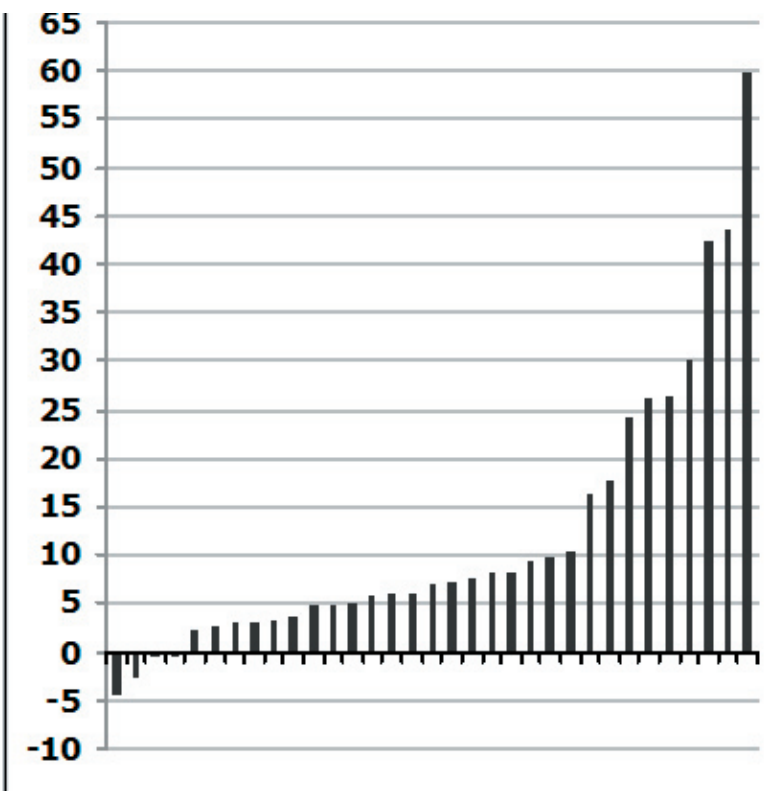

Figura 6. PIC media.

tar un diagnóstico de disfunción del sistema.

La radiología mostró diversos grados de dilatación ventricular en 28 de los 33 casos no arrojando un diagnóstico concluyente en ninguno de ellos dada la ausencia de signos de hidrocefalia activa (edema periependimario o cambios de tamaño respecto a exploraciones previas) (Figura 3). En 5 casos el diagnóstico radiológico fue de ventrículos en hendidura (Figura 4). Uno de los pacientes presentaba una siringomielia asociada a una malformación de Chiari y una dilatación ventricular connatal (Figura 5).
El tiempo de evolución de los síntomas fue muy variable y en ocasiones difícil de definir, puesto que en la mayoría de los casos se trataba de pacientes con quejas crónicas en el contexto de radiologías no sugestivas de hidrocefalia activa, por lo que no se había propuesto ningún tipo de exploración complementaria, limitándose el facultativo a realizar un seguimiento clínico periódico y prescribir tratamientos sintomáticos.

En la tabla 2 se resumen las características clínicas y de monitorización de los pacientes, así como la valoración global del registro.

La duración de la monitorización osciló entre 5 y 136 horas, con una media de 65.24 horas.

La PIC media de los distintos registros (Figura 6) osciló entre 4.41 y $59.6 \mathrm{mmHg}$ con una media total de 12.04 mmHg. La PIC media se situó por debajo de $10 \mathrm{mmHg}$ en 23 de los 33 casos (69.7\%), entre 10 y $15 \mathrm{mmHg}$ en 1 caso $(3 \%)$ y por encima de $15 \mathrm{mmHg}$ en el resto $(27.3 \%)$. Siguiendo exclusivamente el criterio de considerar patológicos los registros con PIC media por encima de $15 \mathrm{mmHg}$ catalogaríamos como tales al $27.3 \%$ de los pacientes de la serie.

Los valores medios de la PIC sistólica fueron de 14.57 $\mathrm{mmHg}$ en tanto que los de la diastólica fueron de 7.02 mmHg. Las diferencias entre PIC sistólica y diastólica oscilaron entre 0,35 y $13,98 \mathrm{mmHg}$ siendo mayor en los casos en los que la PIC media era más elevada.

Durante el día se registraron valores de PIC menores a los nocturnos. La PIC media diurna global de la serie fue de $8,45 \mathrm{mmHg}$ (entre -6.34 y 41.63 ) frente a los $12,49 \mathrm{mmHg}$ (entre -1.1 y 45.2 ) registrados por la noche. Esta diferen-

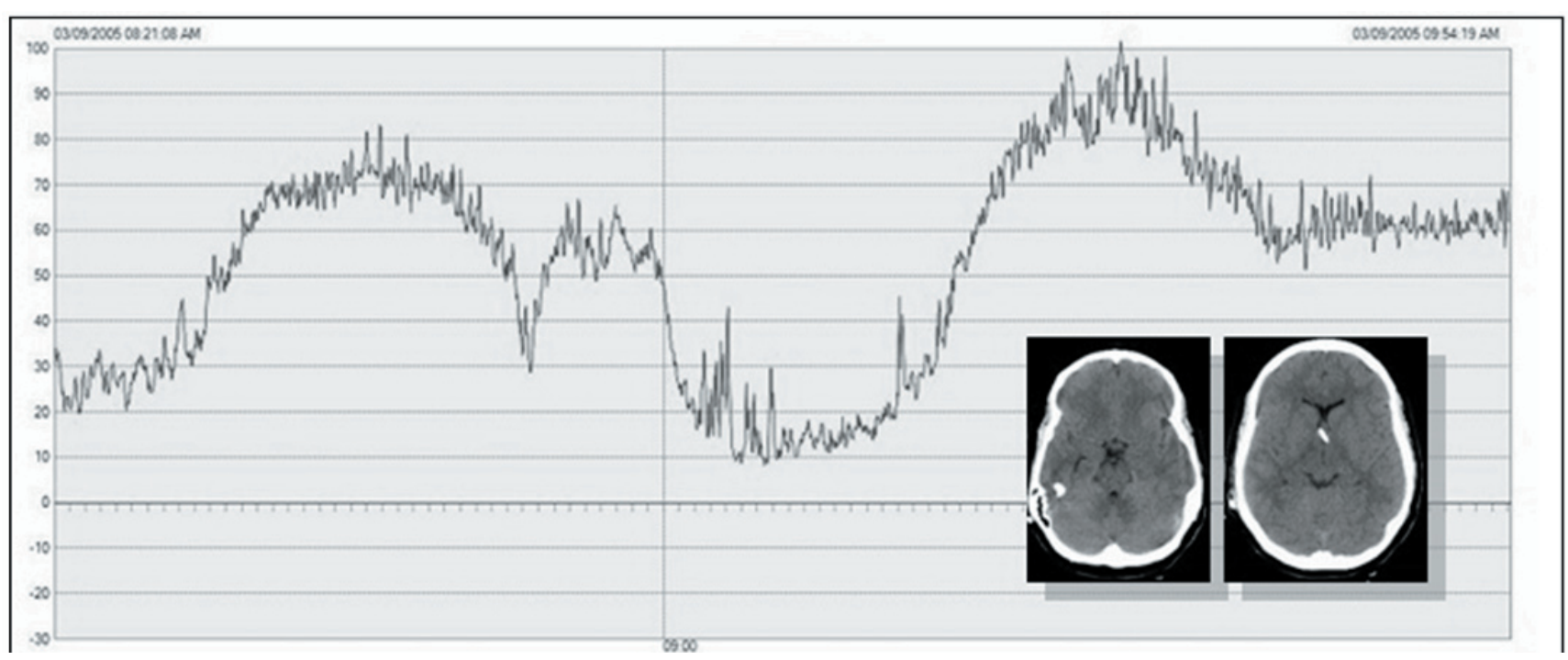

Figura 7. Ondas A con ascenso y descenso más o menos brusco, duración prolongada y gran amplitud. Este paciente, portador de DVP desde el nacimiento, (ID 33) acudió a Urgencias tras sufrir crisis comicial y comenzar con posterioridad con diploplia. Fue dado de alta, reingresando por cefalea. La imagen que acompaña al registro muestra unos ventrículos en hendidura similares a exploraciones previas. PIC media: 43,58 $\mathrm{mmHg}$. 


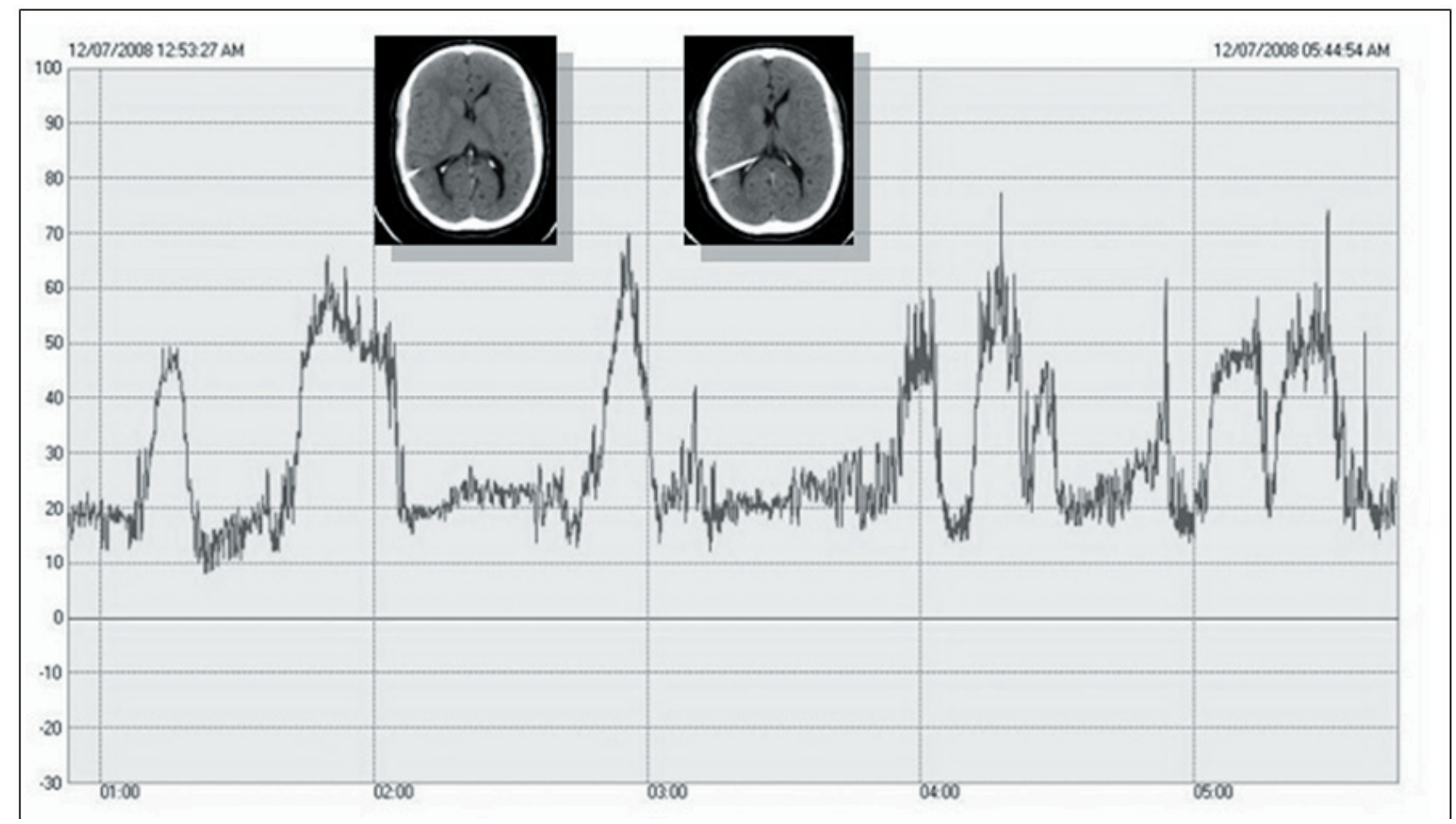

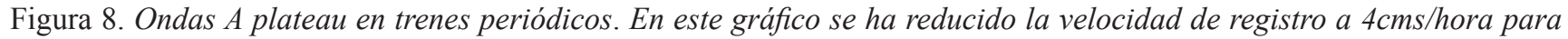
apreciar con mayor nitidez la periodicidad de las ondas plateau. Paciente (ID 22) con DVP connatal, clínica de cefalea de 1 mes de evolución y TAC informado como ventrículos en hendidura sin cambios respecto a estudios previos. PIC media: $16,2 \mathrm{mmHg}$.

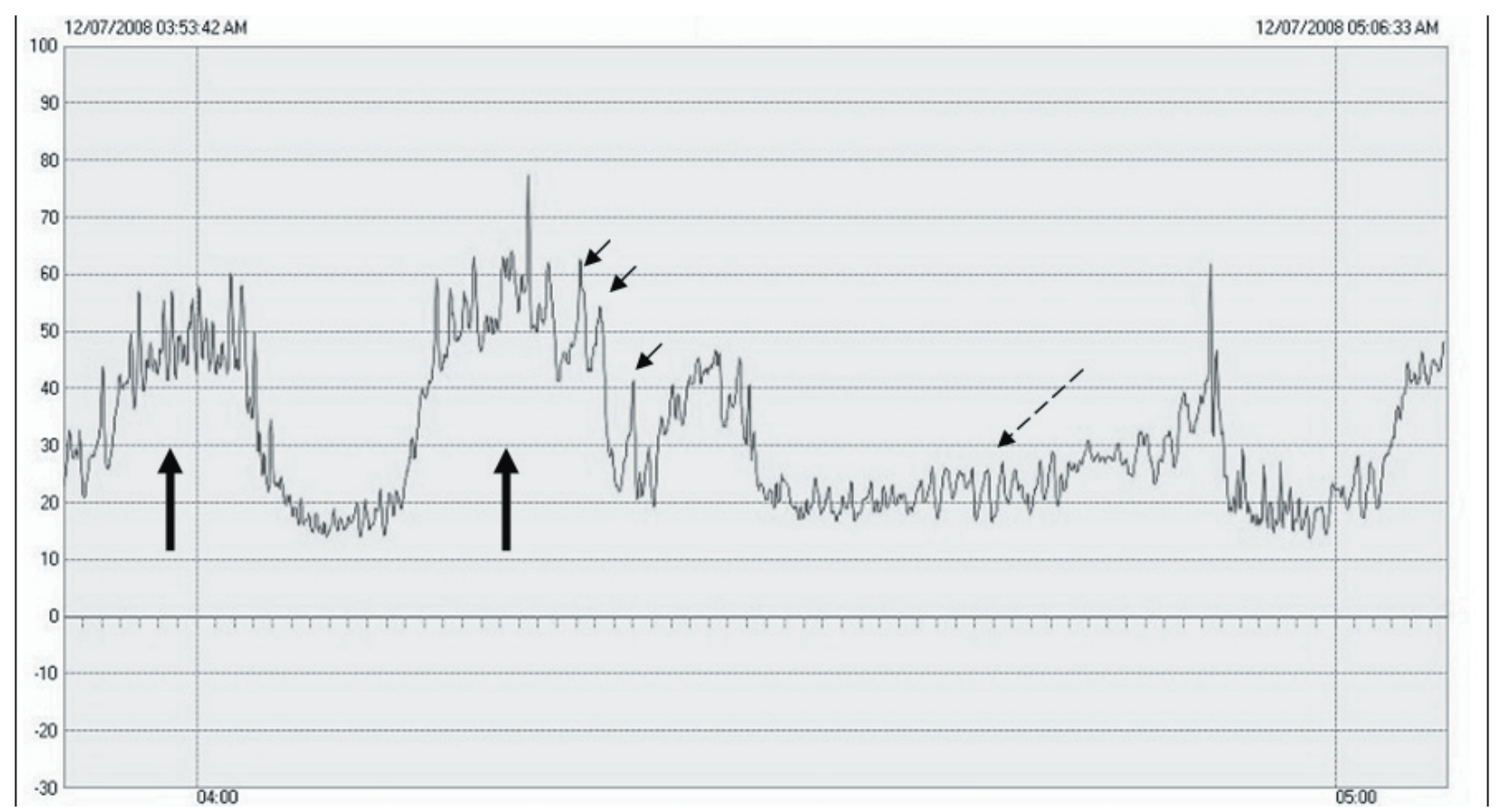

Figura 9. Ondas A plateau (flecha sólida) en trenes sobre las que se superponen ondas B de alta amplitud (flecha fina) y ondas $B$ de baja amplitud (flecha discontinua). Se trata del mismo paciente de la Figura 7.11 (ID 22). PIC media: 16,2 mmHg. 


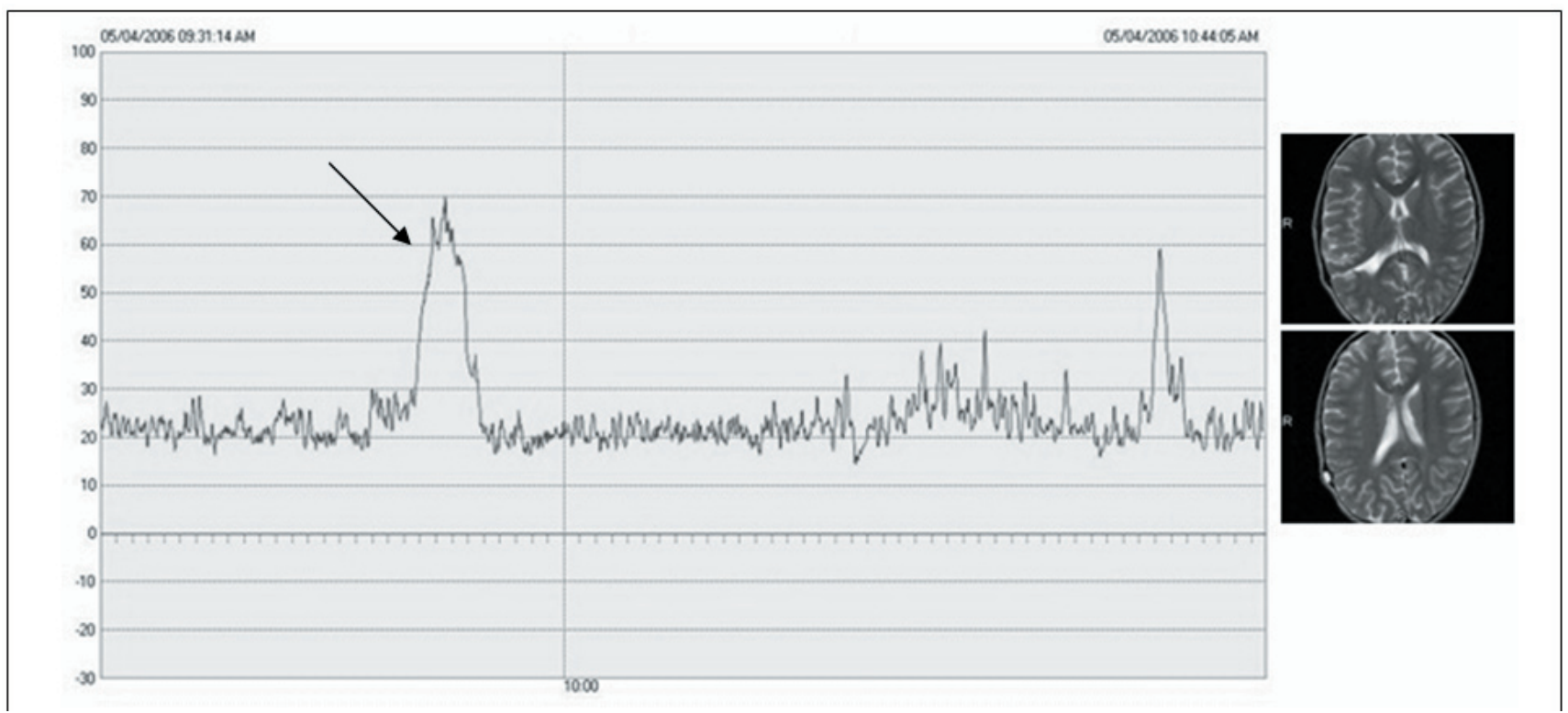

Figura 10. Ondas A preplateau. Puede apreciarse la típica morfología en meseta y descenso súbito, alta amplitud (50-60 mmHg) y duración en torno a 5 minutos. Se trataba de un varón de 9 años (ID 29), portador de DVP desde el nacimiento, que había ingresado en numerosas ocasiones por episodios de cefalea, náuseas y vómitos que cedían en unos días. La RM muestra una reducción del tamaño ventricular. PIC media: $30,15 \mathrm{mmHg}$.

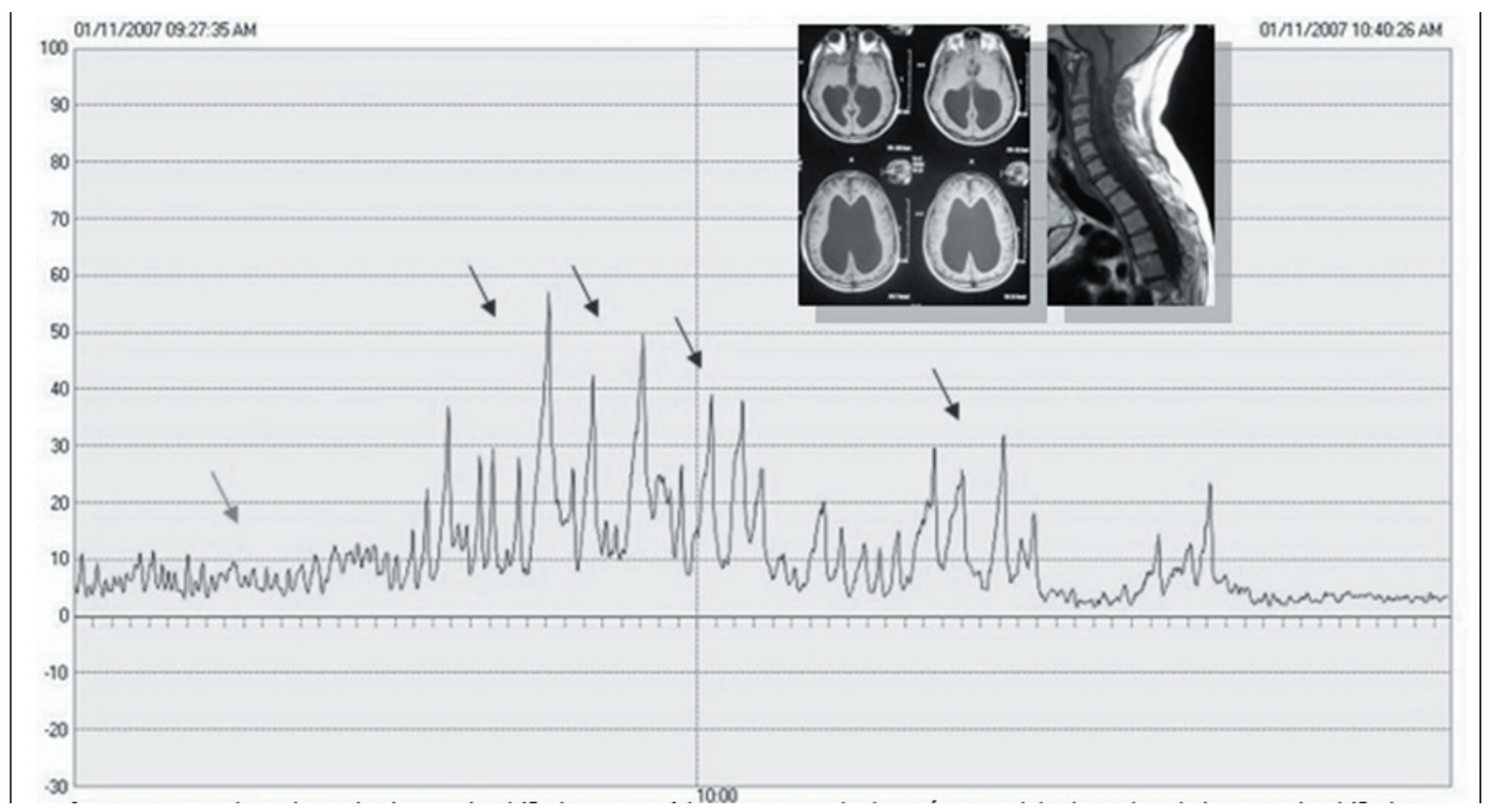

Figura 11. tren de ondas B de alta amplitud (flechas negras)de 32 minutos de duración precedido de ondas de baja amplitud (flecha gris). Este paciente era portador de una DVP por mielomeningocele desde el nacimiento y presentaba un cuadro de cefalea de larga evolución, aumento de su paraparesia y debilidad en manos. La RMN mostraba una dismorfia ventricular con catéter mal alojado (conocida y sin cambios respecto a exploraciones previas) y una siringomielia cérvicodorsal. PIC media: $5.04 \mathrm{mmHg}$ 


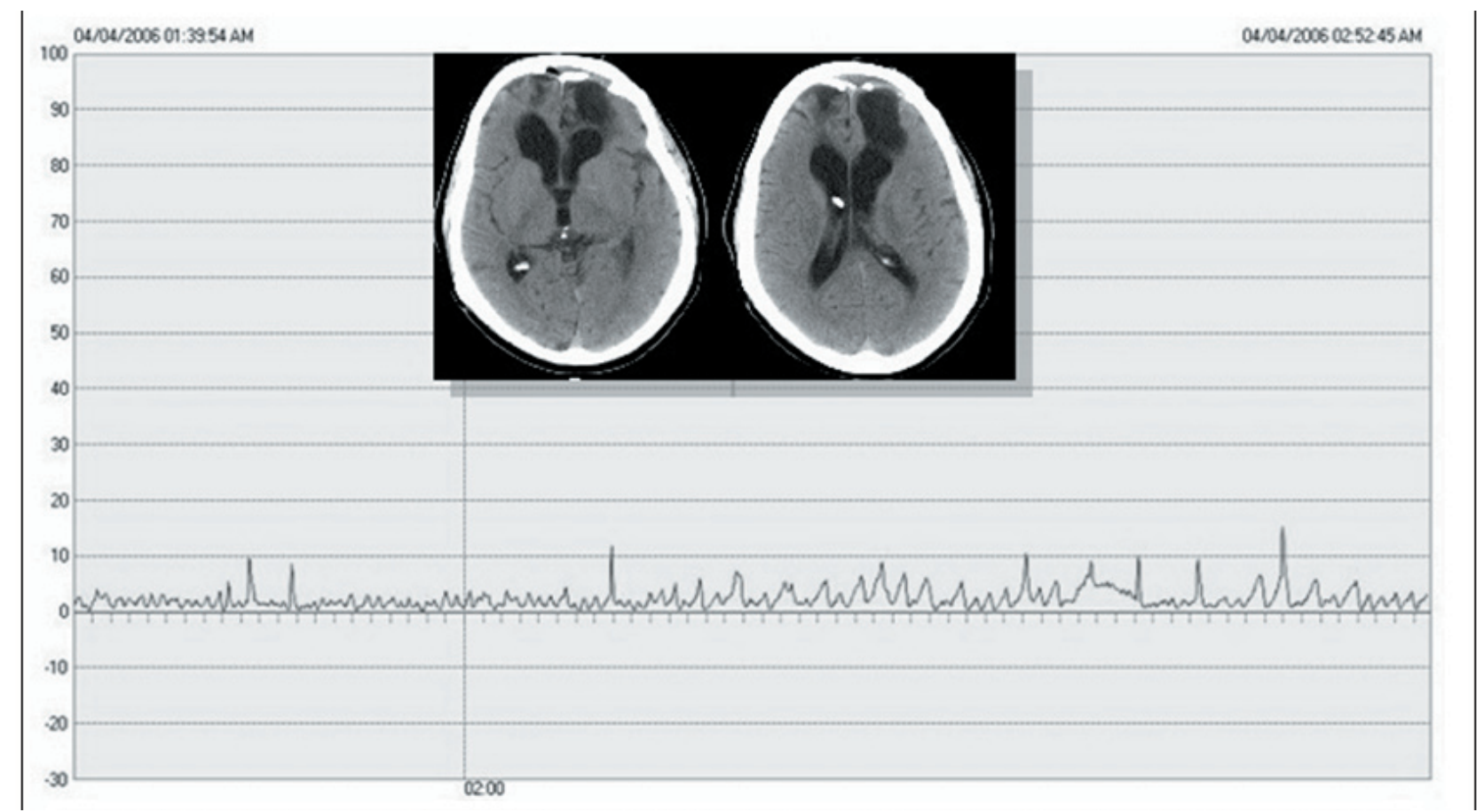

Figura 12. ondas B nocturna de baja amplitud (menor de $10 \mathrm{mmHg}$ ). Paciente (ID 28) con hidrocefalia postraumática y derivación de 8 meses de evolución con cefaleas y deterioro cognitivo progresivo, al que se le había sustituido derivación depresión media por otra de baja presión. La TAC mostraba dilatación ventricular sin signos de actividad junto a zonas de encefalomalacia.

cia también se aprecia en los valores de la PIC sistólica y diastólica nocturnas que fueron de $15,71 \mathrm{mmHg}$ y 9.17 $\mathrm{mmHg}$ (frente a los 14.57 y 7.02 anteriormente referidos). Atendiendo a los valores de la PIC media nocturna, el 56\% de los pacientes presentaron presiones inferiores a los 10 $\mathrm{mmHg}, 22 \%$ presiones entre 10 y $15 \mathrm{mmHg}$ y otro $22 \%$ por encima de $15 \mathrm{mmHg}$.

Las ondas patológicas fueron detectadas en 25 casos (75\%). El resto de los pacientes no presentaron ondas A ni B.

Las ondas A fueron visualizadas en 11 casos $(33,33 \%)$
(Figura 7 y 8). Todos los casos que en los que se registraron ondas A en el registro presentaron además ondas B que fueron siempre de alta amplitud. Los pacientes de este grupo presentaron en su mayoría los dos tipos de ondas A descritos, tanto ondas plateau como preplateau (Figuras 9 y 10 ), detectándose sólo ondas preplateau en un $27 \%$ de estos sujetos. En varios casos se detectaron trenes de ondas A periódicas, tanto en su formato preplateau como plateau (Figuras 7, 8 y 9). La PIC media de estos pacientes fue muy elevada situándose en $26.78 \mathrm{mmHg}$, siendo aún mayor si excluimos del cálculo los dos casos que no presentaron

Tabla 3

Tipos de onda y combinaciones

\begin{tabular}{|c|c|c|c|c|c|}
\hline \multicolumn{2}{|c|}{ Ondas A } & \multicolumn{2}{c|}{ OndasB } & \multirow{2}{*}{ \% pacientes } & PIC media \\
\cline { 1 - 3 } Plateau & Preplateau & Alta amplitud & Baja amplitud & \\
\hline+ & + & + & + & $24 \%$ & 30.76 \\
- & + & + & + & $9 \%$ & 16.16 \\
- & - & + & + & $15 \%$ & 8.94 \\
- & - & - & + & $27 \%$ & 3.40 \\
- & - & - & - & $25 \%$ & 3.46 \\
\hline
\end{tabular}




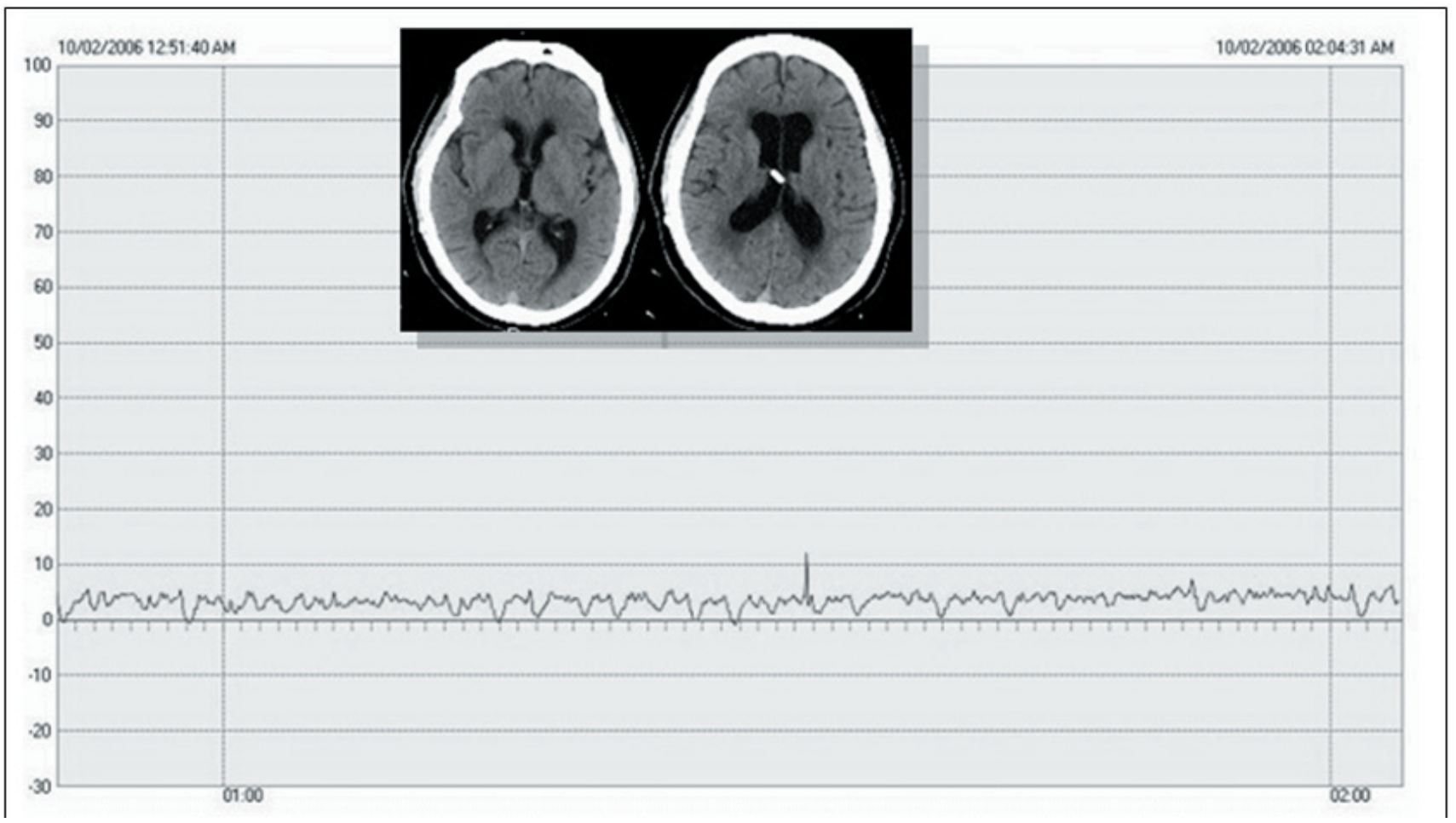

Figura 13. Imagen de registro considerado como normal. Se parecían pequeñas oscilaciones de la línea base sin apreciarse ondas patológicas. Se trataba de un paciente (ID 23) portador de una DVP por hidrocefalia postraumática con cuadro de cefalea y deterioro cognitivo de meses de evolución. La TAC mostraba una dilatación ventricular con catéter correctamente alojado.

ondas plateau sino solo preplateau, en cuyo caso la PIC media se sitúa en $30.69 \mathrm{mmHg}$.

Las ondas B se detectaron en 25 de los 33 casos $(75.75 \%)$. En el $60 \%$ de los pacientes que presentaron ondas B predominaron las ondas B de alta amplitud (Figura 11) y el $78 \%$ de éstos asociaron además ondas tipo A (Figuras 9 y 10). Ninguno de los pacientes en los que predominaban las ondas B de baja amplitud presentaron ondas A y tan sólo uno de ellos presentó además ondas $\mathrm{B}$ de alta amplitud aisladas. El porcentaje de ondas B fue superior al 20\% del trazado nocturno en todos aquellos pacientes que presentaron ondas B de alta amplitud. Entre los que predominaban las ondas B de baja amplitud (Figura 12), solo el 40\% de los pacientes las presentaban en más del $20 \%$ del trazado nocturno. La PIC media de los pacientes que presentaron ondas B asociadas a ondas plateau fue de $30.76 \mathrm{mmHg}$, en tanto que los que lo hicieron asociados a las preplateau fue de $16.16 \mathrm{mmHg}$. Los pacientes que presentaron ondas B de alta amplitud no asociadas a ondas A tuvieron una PIC media de $8.94 \mathrm{mmHg}$ y los que presentaron sólo ondas B de baja amplitud una PIC media de $3.40 \mathrm{mmHg}$. Las diversas combinaciones de ondas de estos pacientes se recogen en la Tabla 3, así como las PIC medias de cada grupo.

En 25\% de los pacientes el registro no demostró ondas patológicas de ningún tipo (Figura 13).

Atendiendo a los criterios de presencia de ondas patológicas y porcentaje de las mismas sobre el trazado nocturno, el $51 \%$ de los pacientes tuvieron registros que fueron considerados anormales.

Teniendo solo como criterio diagnóstico la cifra de PIC media, solo el $27.3 \%$ de los pacientes presentó cifras por encima de $15 \mathrm{mmHg}$. Esto quiere decir que siguiendo este solo criterio diagnóstico serían considerados normales cerca de la mitad de los pacientes que serían diagnosticados

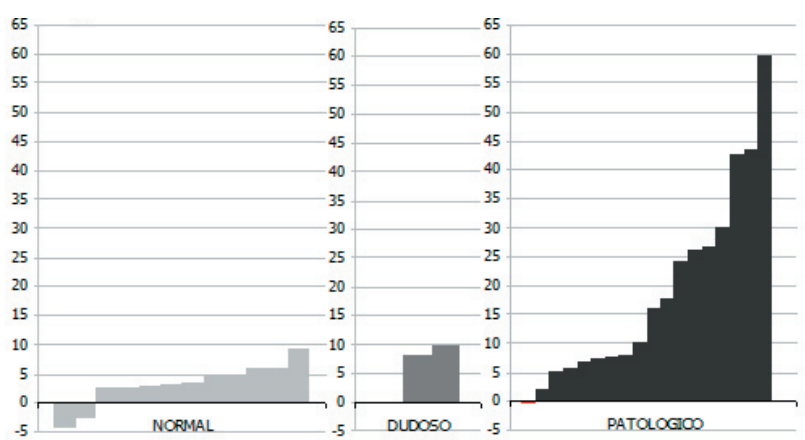

Figura 14. Valores de PIC media y valoración de registros según análisis morfológico. 


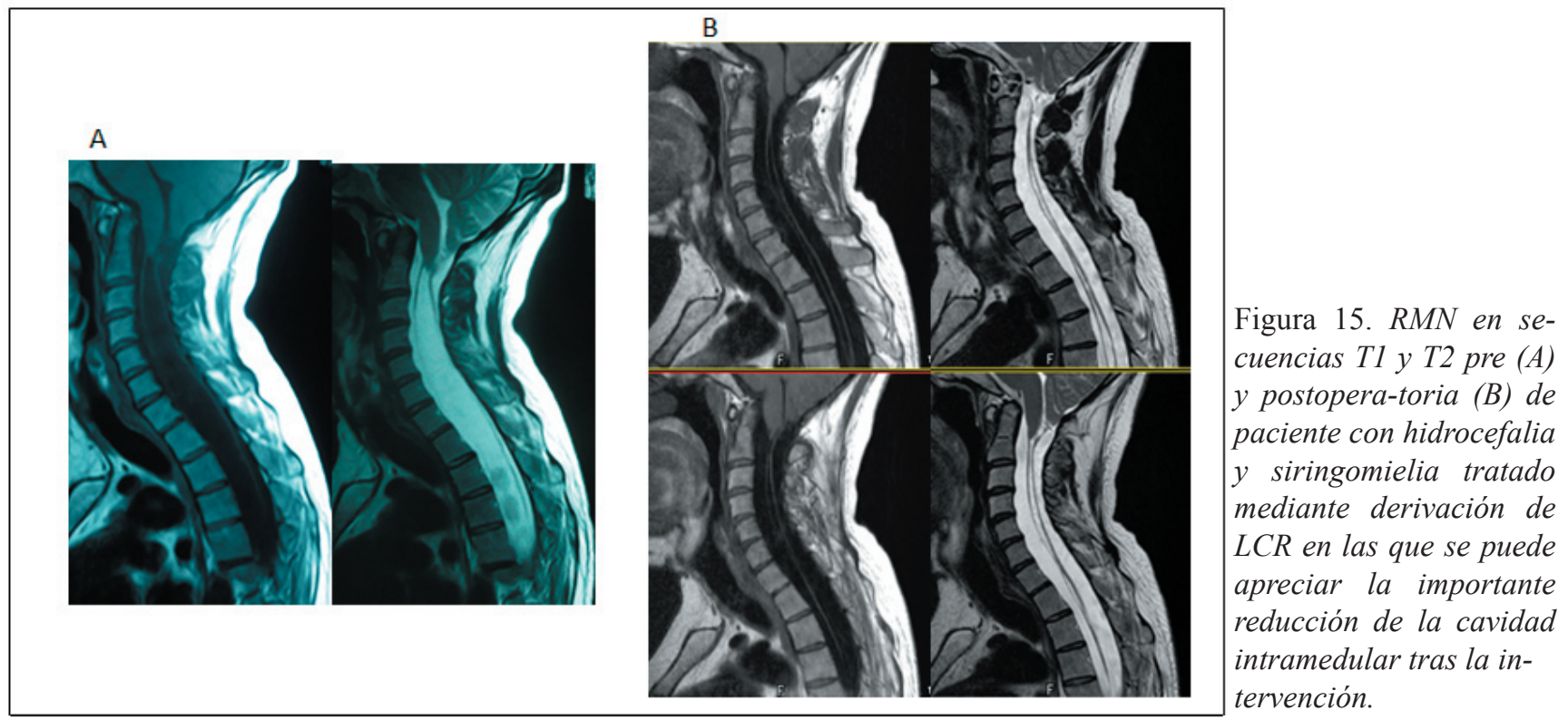

de disfunción valvular con criterios morfológicos del registro. En la Figura 14 se representan los valores medios de PIC en cada uno de los diagnósticos realizados tras valorar morfológicamente los registros.

Los síntomas que mayor relación tienen con los registros patológicos son la cefalea y el papiledema. La presencia de deterioro visual en pacientes portadores de una DVP no siempre se relaciona con registros patológicos en esta serie, de manera que lo está en el $40 \%$ de ellos. Sin embargo, cuando lo hace se asocia a papiledema en todos los casos y presenta registros muy patológicos con presencia de ondas A y B y PIC media global en torno a los $51 \mathrm{mmHg}$. La cefalea se asoció a registros patológicos en el $61 \%$ de los casos, siendo la PIC media de ellos de 21,5 mmHg.

No hubo ningún tipo de complicación relacionada con el procedimiento de monitorización, registrándose tan sólo una autorretirada del sensor antes de completar el periodo establecido de registro, sin consecuencias ya que el trazado fue lo suficientemente significativo.

En todos los casos en los que el registro fue catalogado de patológico se procedió a una revisión del sistema derivativo, salvo uno de los casos por negativa familiar al ser un niño de 9 años, aún tratándose de un cuadro de hipertensión intracraneal grave y clínica de cefaleas episódicas. Este caso reingresó meses más tarde con un importante papiledema y grave deterioro de agudeza visual, procediéndose entonces a la revisión del sistema. En los casos considerados no patológicos y dudosos se optó por la abstención terapéutica.

En los casos en los que se realizó revisión del sistema derivativo los procedimientos utilizados fueron diversos:
- Derivación lumbo-peritoneal o ventrículo-peritoneal con dispositivos antigravitatorios (ajustable o no) en los casos de ventrículos en hendidura e hipertensión intracraneal.

- Sustitución del sistema derivativo por otro de menor presión: cambio de derivaciones de presión media por válvulas de baja presión o de éstas por tubos libres, acompañadas siempre de sistemas antigravitatorios.

- Sustitución por derivaciones programables con dispositivos antisifón en casos de grandes ventriculomegalias.

No se produjeron complicaciones precoces relacionadas con las intervenciones quirúrgicas, registrándose un hematoma subdural crónico asintomático en un paciente con gran ventriculomegalia y una derivación programable que se resolvió mediante el aumento de presión de apertura de la válvula.

A los 6 meses todos los pacientes que habían sido sometidos a revisión valvular habían experimentado mejoría de sus síntomas respecto a su situación preoperatoria. Los dos pacientes que presentaban importante deterioro de la agudeza visual previo al tratamiento, mejoraron de la cefalea pero no recuperaron la función visual. El paciente que presentaba la siringomielia presentó una resolución radiológica de la misma con la consiguiente mejoría clínica (Fig 15). Desde el punto de vista radiológico la respuesta al tratamiento fue variable y mientras se apreciaron reducciones en el tamaño ventricular en algunos de los pacientes (Fig. 16), en otros el mismo se mantenía sin cambios respecto a las exploraciones prequirúrgicas. Las puntuación en la escala de Karnofski mejoró sustancialmente en casi todos los pacientes como se refleja en la fig. 17. 

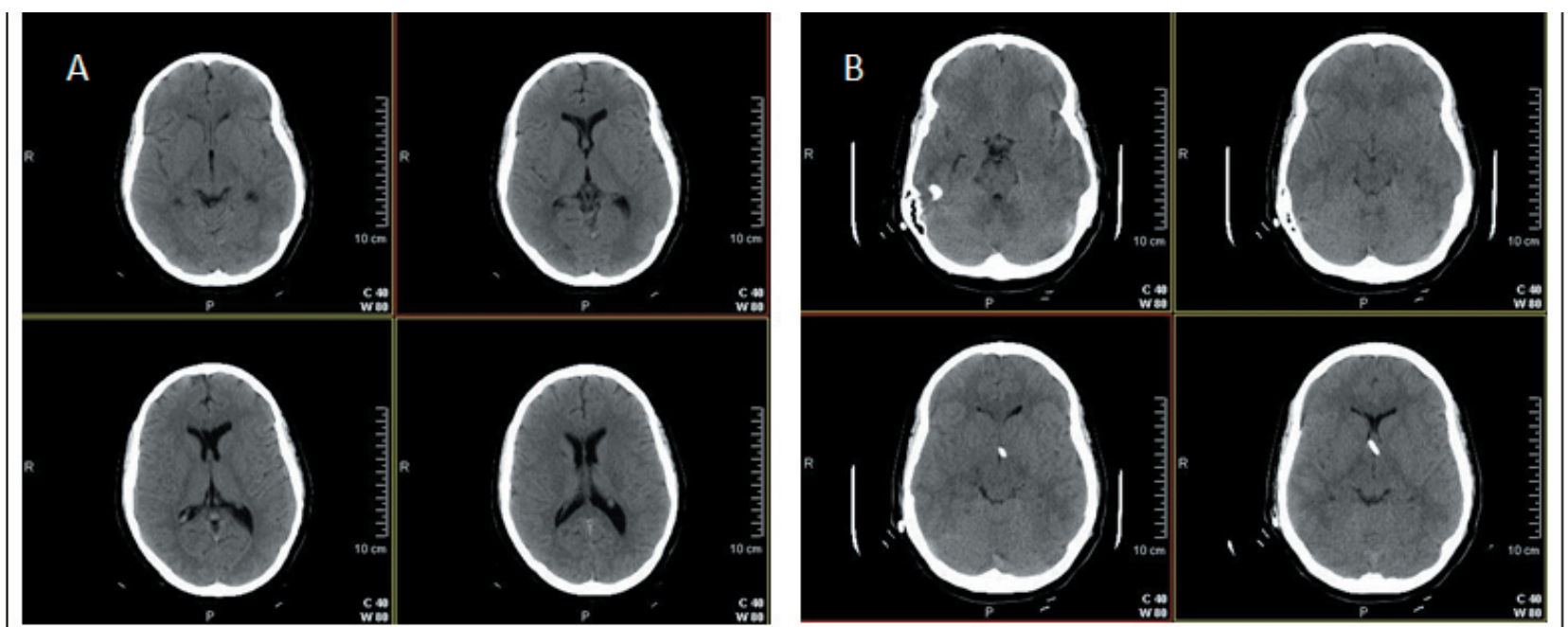

Figura 16. TAC pre (A) y postquirúrgico (B) de un paciente portador de DVP connatal y cuadro de cefalea con registro muy patológico tratado con derivación lumboperitoneal. Se puede apreciar una reducción del tamaño ventricular. La primera exploración no obstante fue informada como normal. Desde el punto de vista clínico se produjo la resolución de la clínica.

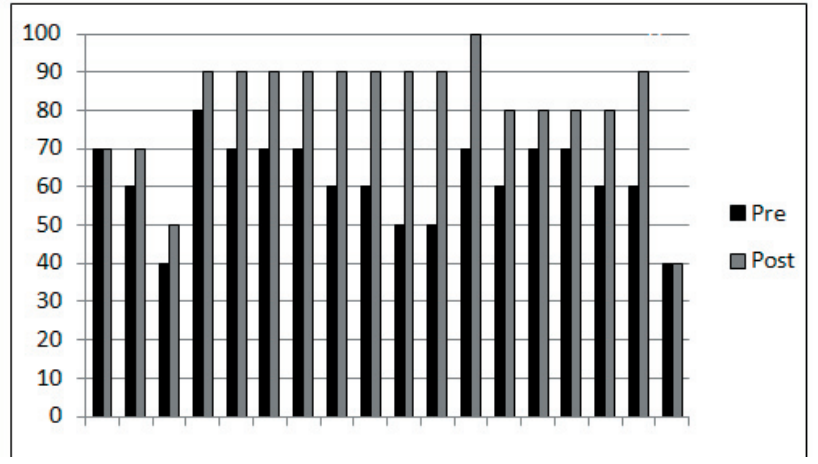

Figura 17. Puntuaciones en la escala de Karnofski pre y postoperatorias de los pacientes tratados tras la monitorización de la PIC.

\section{Discusión}

En los pacientes con derivaciones de LCR la presencia de síntomas habitualmente relacionados con hipertensión intracraneal (cefalea, náuseas, vómitos o somnolencia) no siempre son atribuibles a esta causa ${ }^{1,9-11,13,18,22-24}$, y por tanto el diagnóstico de disfunción valvular basado en criterios clínicos resulta muy difícil. Desde el punto de vista radiológico podemos distinguir entre los pacientes que presentan diversos grados de dilatación ventricular en el contexto de dismorfias ventriculares de larga evolución y los que tienen ventrículos de los denominados en hendidura. En nuestra serie, en ninguno de los casos recogidos el diagnóstico de disfunción valvular se realizó a partir de los resultados radiológicos, que no mostraron cambios llamativos respecto a estudios previos. En la literatura existen estudios que confirman que no se puede correlacionar el tamaño ventricular o los cambios del mismo con las cifras de PIC, por lo que el diagnóstico de disfunción valvular basada en este dato debe ser hecho de forma muy caute$\operatorname{losa}^{8}$. En el caso de los ventrículos en hendidura la situación se complica aún más, habiéndose descrito hasta cinco situaciones fisiopatológicas diferentes con distintas cifras de presión intracraneal, que van desde hipotensiones a severas hipertensiones, en contextos clínicos y radiológicos similares $^{18,22-24}$.

Por tanto, el dictamen de disfunción valvular suele requerir la utilización de otros recursos diagnósticos, especialmente en los casos de disfunción crónica (los agudos suelen tener una clínica y una radiología altamente sugestiva). En la literatura se han descrito algunos métodos relacionados con ellos, como la realización de test de infusión ${ }^{19}$, con resultados positivos. Sin embargo, estos test precisan de la manipulación del reservorio, con los riesgos de deterioro del mismo que ello conlleva y evalúan el funcionamiento del shunt sin informar acerca de la situación y la dinámica de la PIC en el paciente.

La monitorización de la PIC en pacientes con sospecha de disfunción valvular es un método descrito en la literatura en numerosos trabajos, si bien es cierto que la metodología en la valoración de los registros varía de unos a otros. Constituye un método relativamente sencillo, seguro ${ }^{14,16,17,21}$ y que aporta una valiosa información. En algunos trabajos, se utiliza el registro de PIC para evaluar fundamentalmente la presión media, sin atender excesivamente a la morfología del trazado ${ }^{13}$. En la mayoría sin embargo, y especialmente en los trabajos más recientes, se atribuye más valor a la presencia de ondas patológicas que al valor medio de la PIC ${ }^{4,20}$. En algunos de ellos no se evalúa adecuadamente la presencia de ondas B y los criterios para considerar anormal un registro varían de unos autores a otros. En los 


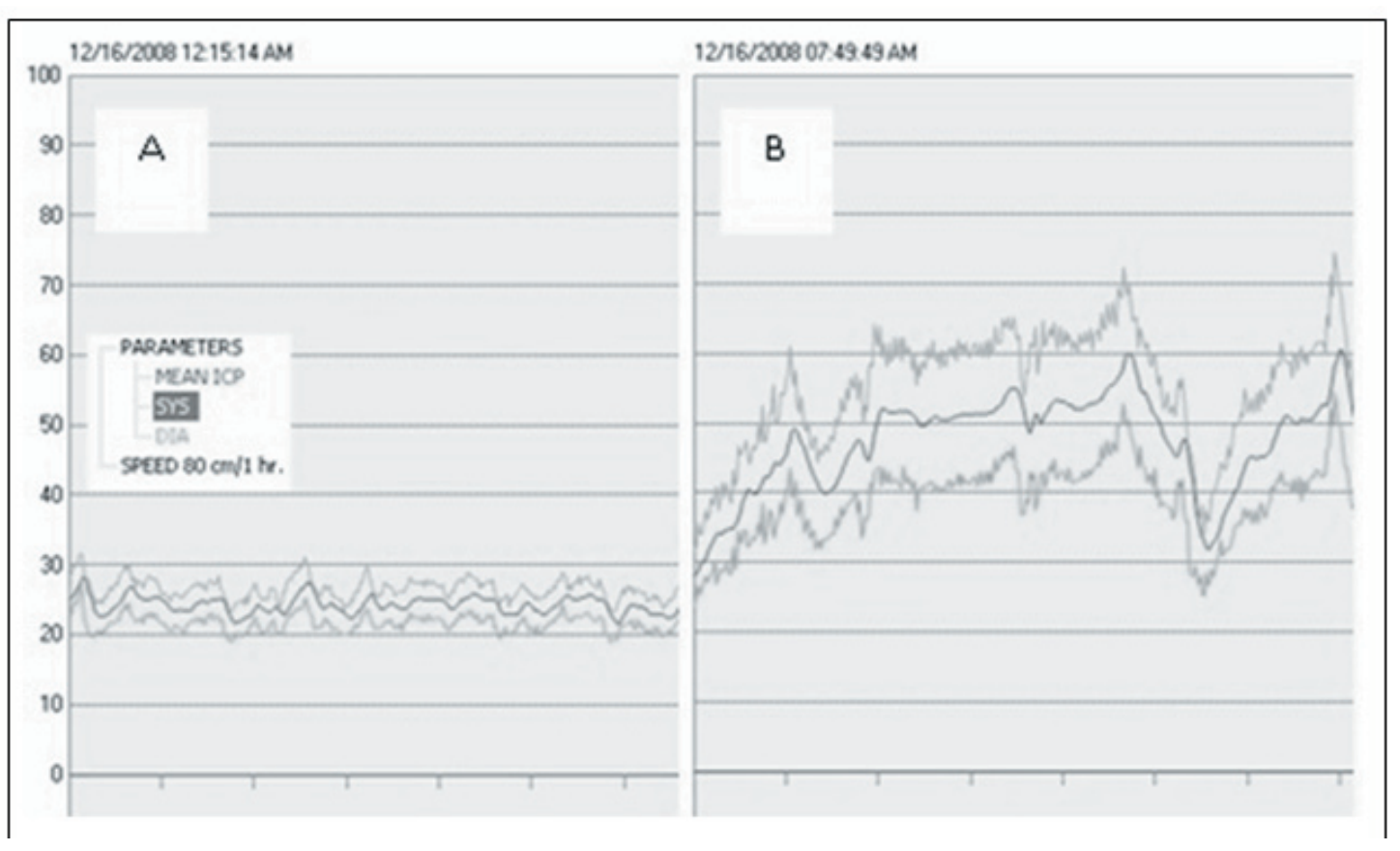

Figura 18. Ejemplo de dos fragmentos de trazado de PIC en los que puede apreciarse la diferencia entre el componente sistólico (trazo superior) y el diastólico (trazo inferior) de la PIC en relación a la PIC media (trazo intermedio) en el mismo paciente. El fragmento A, con PIC media en torno a $25 \mathrm{mmHg}$ presenta diferencias de unos $5 \mathrm{mmHg}$ en tanto que el fragmento B, cuya PIC media es de unos $45 \mathrm{mmHg}$, presenta diferencias cercanas a $20 \mathrm{mmHg}$.

últimos años se ha popularizado el análisis computarizado de los registros, mediante patrones que permiten reconocer las ondas B y su amplitud ${ }^{9,10,12}$. En general, se admite que un elevado porcentaje de ondas B en el registro es predictor positivo de una buena respuesta a la derivación ${ }^{9,26}$.

En la serie analizada destacan pacientes con sintomatología relativamente anodina (cefaleas episódicas de larga evolución) y exámenes radiológicos superponibles a exploraciones previas, en los que se detectan cifras de PIC muy elevadas con presencia de ondas plateau. Habitualmente son pacientes en seguimiento ambulatorio y con visitas repetidas a Urgencias en los que no se llega a un diagnóstico de disfunción valvular basado exclusivamente en estos criterios. Desde el punto de vista clínico, la presencia de diplopia y papiledema se correlaciona con registros muy patológicos y cifras medias de PIC muy elevadas en la mayoría de los casos. Desgraciadamente, la funduscopia no es práctica habitual en los servicios de urgencias y algunos de estos pacientes han sido estudiados cuando ha comenzado a existir un déficit visual. La cefalea es un síntoma frecuente en pacientes portadores de derivaciones de LCR y en la serie presentada aparecía en todos los sujetos estudiados excepto en aquellos casos de hidrocefalia crónica tratados con derivación y mala evolución posterior. Sin embargo, aún siendo un síntoma frecuente, es inespecífico y no se correlaciona de forma significativa con registros anormales, estando presente también en pacientes con trazados normales. Los pacientes portadores de derivaciones desde el nacimiento con sospecha de disfunción constituyen el paradigma de esta situación: pacientes con dismorfias ventriculares y quejas de larga evolución más o menos tolerables no atribuidas a problemas valvulares a los que tras registro de PIC se diagnostican de disfunción valvular, mejorando de sus síntomas tras la revisión del sistema. Los ventrículos en hendidura constituyen asimismo un subgrupo interesante. Los registros patológicos en estos pacientes son extremadamente anormales con cifras de PIC media muy elevada y presencia de ondas anormales, tanto A como B de muy alta amplitud. Los pacientes portadores de sistemas desde el nacimiento presentaron con mayor frecuencia que el resto registros patológicos. Por tanto, la edad de la válvula en el momento del registro fue notablemente superior en aquellos pacientes en los que el mismo terminó siendo patológico que en los que el registro fue normal (media de 109 meses frente a 44).

En la serie presentada y basados exclusivamente en criterios de PIC media, habrían sido diagnosticados como disfunciones valvulares algo más del $27 \%$ de los sujetos estudiados, cifra muy lejana al $51 \%$ de pacientes a los que finalmente se les adjudicó esté diagnóstico tras el análisis morfológico del registro. Todos los pacientes con cifras de PIC por encima de los $15 \mathrm{mmHg}$ presentaron registros anor- 
males desde el punto de vista morfológico, pero un $29 \%$ con cifras inferiores a esta cifra tuvieron registros patológicos. Podemos decir que el valor predictivo positivo de este criterio es muy alto (100\%) pero el negativo no (40\%), dejando muchos pacientes por diagnosticar. Un $40 \%$ de los pacientes con cifras de PIC media $<15 \mathrm{mmHg}$ presentaron ondas B de alta amplitud, como los casos de los pacientes ID17 y ID24 en los que, con cifras medias de PIC de 8.12 y $5.07 \mathrm{mmHg}$ respectivamente, se apreciaron ondas B de alta amplitud en gran parte del registro, con cifras de PIC que llegaban a rebasar los 40 y $50 \mathrm{mmHg}$ (Fig. 11). Por lo tanto es imprescindible un análisis morfológico del trazado para clasificar correctamente a estos pacientes. La presencia de ondas B de alta amplitud se correlaciona en esta serie con registros patológicos en todos los casos y cuando aparecen son visibles en gran parte del registro nocturno, yendo a menudo asociadas a ondas plateau y preplateau (Figs. 9 y 10), así como a ondas B de baja amplitud.

La presencia de ondas B de baja amplitud no asociadas a otro tipo de ondas es una situación descrita en esta serie en algo más de una cuarta parte de los registros (25\%). Aparecen siempre en el contexto de PIC media baja y la valoración final del registro en estos sujetos fue normal o planteó dudas. La presencia de ondas B de baja amplitud en un porcentaje muy elevado del registro nocturno fue considerado patológico en ausencia de ondas B de alta amplitud, hecho que ocurrió en 3 de los 9 casos en los que se evidenciaron (Fig 12). En estos casos el porcentaje de ondas estaba por encima del $30 \%$ del trazado, obteniéndose mejoría clínica tras la revisión valvular en todos ellos.

La diferencia entre la PIC sistólica y diastólica es un dato indirecto de la complianza cerebral, es decir, de la capacidad del parénquima para amortiguar los aumentos de presión. En situaciones de baja complianza, pequeños aumentos de volumen intracraneal generan aumentos importantes de la presión, siguiendo la conocida curva de presión-volumen. La disminución de la complianza cerebral se ha demostrado tanto en pacientes con trauma craneal como en sujetos con hidrocefalia. En los primeros parece más relacionada con los cambios en el volumen sanguíneo cerebral y en los segundos con las cifras de PIC media $^{2}$. Algunos autores han demostrado que la respuesta positiva a la derivación es superior en HCA en aquellos pacientes en los que la amplitud del pulso de la PIC es mayor ${ }^{7,3}$, así como que dicha amplitud se reduce tras la colocación de dispositivos de derivación de $\mathrm{LCR}^{6,3}$. En los pacientes de esta serie se aprecia cómo el aumento de la PIC media se correlaciona con mayores diferencias entre los componentes sistólico y diastólico de la PIC. En la Fig. 18 se muestran dos fragmentos de un registro de PIC realizado al mismo paciente con PIC medias diferentes donde puede observarse cómo la diferencia entre los valores del componente sistólico y diastólico es mayor cuanto mayor es la PIC media.

Dentro del grupo de las disfunciones valvulares llama especialmente la atención el grupo de pacientes con ventrículos en hendidura. La mayoría de los casos registrados se presentaban con síntomas visuales y mostraban cifras de PIC media muy elevadas con trazados muy anormales, repletos de ondas B de alta amplitud y ondas B. Es especialmente llamativo el caso ID29, que ingresa con cuadro de cefalea y vómitos. Previamente se habían detectado ingresos repetidos por la misma sintomatología a intervalos de varios meses, tratados sintomáticamente con resolución de la clínica. Durante uno de esos ingresos se monitoriza la PIC y se obtiene un registro muy anormal con cifras de PIC media en torno a los $30 \mathrm{mmHg}$, por lo que se recomienda la revisión valvular. La familia se niega a ello y tras mejoría clínica solicitan alta voluntaria. Reingresa doce meses después con clínica similar a la que se añade un deterioro visual grave por papiledema bilateral. Tras la revisión valvular se normalizan las cifras de PIC pero el paciente no recupera visión. En este grupo de pacientes, pueden estar presentes cifras de PIC muy elevadas en ausencia de sintomatología o con clínica relativamente anodina, lo que puede condicionar deterioros clínicos silentes al tratarse de niños, y en ocasiones irreversibles, por lo que la monitorización de la PIC constituye una herramienta de extraordinaria utilidad $\mathrm{d}^{5,25}$

\section{Conclusiones}

En pacientes con sospecha de disfunción valvular en los que ni la clínica, ni la exploración, ni las pruebas radiológicas sean suficientes para confirmar o descartar un diagnóstico, la monitorización de la PIC constituye un método seguro y fiable para evaluar la situación de la derivación que debe ser utilizado. El análisis cuantitativo exclusivamente no es suficiente siendo preciso un análisis morfológico del trazado que permita identificar la presencia de ondas anormales, por lo que es preciso dotarse de sistemas de captura y representación de los datos que permitan la adecuada evaluación del registro. Las ondas A acontecen siempre en el contexto de PIC medias elevadas y por lo tanto son patológicas. Las ondas B de alta amplitud pueden aparecer en registros con PIC medias normales, pero son siempre expresión de situaciones patológicas. Las ondas B de baja amplitud, especialmente cuando aparecen de manera aislada, no se corresponden, desde nuestro punto de vista, con situaciones de disfunción valvular.

\section{Bibliografía}

1. Abbott, R., Epstein, F.J., Wisoff, J.H.: Chronic headache associated with a functioning shunt: Usefulness of pressure monitoring. Neurosurgery. 1991; 28: 72,6; discussion 76-77. 
2. Carrera, E., Kim, D.J., Castellani, G., Zweifel, C., Czosnyka, Z., Kasparowicz, M., et al.: What shapes pulse amplitude of intracranial pressure? J Neurotrauma. 2010; 27: 317-324.

3. Czosnyka, M., Czosnyka, Z., Keong, N., Lavinio, A., Smielewski, P., Momjian, S., et al.: Pulse pressure waveform in hydrocephalus: What it is and what it isn't. Neurosurg Focus. 2007; 22: E2.

4. Czosnyka, M., Pickard, J.D.: Monitoring and interpretation of intracranial pressure. J Neurol Neurosurg Psychiatry. 2004; 75: 813-821.

5. Dahlerup, B., Gjerris, F., Harmsen, A., Sorensen, P.S.: Severe headache as the only symptom of long-standing shunt dysfunction in hydrocephalic children with normal or slit ventricles revealed by computed tomography. Childs Nerv Syst. 1985;1: 49-52.

6. Eide, P.K., Sorteberg, W.: Diagnostic intracranial pressure monitoring and surgical management in idiopathic normal pressure hydrocephalus: A 6-year review of 214 patients. Neurosurgery. 2010; 66: 80-91.

7. Eide PK. Intracranial pressure parameters in idiopathic normal pressure hydrocephalus patients treated with ventriculo-peritoneal shunts. Acta Neurochir (Wien). 2006; 148: 21,9; discussion 29

8. Eide, P.K.: The relationship between intracranial pressure and size of cerebral ventricles assessed by computed tomography. Acta Neurochir (Wien). 2003; 145: 171,9; discussion 179.

9. Eide, P.K.: Quantitative analysis of continuous intracranial pressure recordings in symptomatic patients with extracranial shunts. J Neurol Neurosurg Psychiatry. 2003; 74: 231-237.

10. Eide, P.K., Due-Tonnessen, B., Helseth, E., Lundar, T.: Differences in quantitative characteristics of intracranial pressure in hydrocephalic children treated surgically or conservatively. Pediatr Neurosurg. 2002; 36: 304-313.

11. Eide, P.K., Due-Tonnessen, B., Helseth, E., Lundar, T.: Assessment of intracranial pressure volume relationships in childhood: The lumbar infusion test versus intracranial pressure monitoring. Childs Nerv Syst. 2001; 17: 382-390.

12. Eklund, A., Agren-Wilsson, A., Andersson, N., Bergenheim, A.T., Koskinen, L.O., Malm, J.: Two computerized methods used to analyze intracranial pressure B waves: Comparison with traditional visual interpretation. J Neurosurg. 2001; 94: 392-396.

13. Fouyas, I.P., Casey, A.T., Thompson, D., Harkness, W.F., Hayward, R.D.: Use of intracranial pressure monitoring in the management of childhood hydrocephalus and shuntrelated problems. Neurosurgery. 1996; 38: 726,31; discussion 731-2.

14. Gelabert-Gonzalez, M., Ginesta-Galan, V., SernamitoGarcia, R., Allut, A.G., Bandin-Dieguez, J., Rumbo, R.M.: The camino intracranial pressure device in clinical practice. assessment in a 1000 cases. Acta Neurochir (Wien). 2006; 148: 435-441.

15. Lundberg, N.: Monitoring of intracranial pressure. Proc R Soc Med. 1972; 65: 19-22.

16. Martinez-Manas, R.M., Santamarta, D., de Campos, J.M., Ferrer, E.: Camino intracranial pressure monitor: Prospective study of accuracy and complications. J Neurol Neurosurg Psychiatry. 2000; 69: 82-86.

17. Munch, E., Weigel, R., Schmiedek, P., Schurer, L.: The camino intracranial pressure device in clinical practice: Reliability, handling characteristics and complications. Acta Neurochir (Wien). 1998; 140: 1113,9; discussion 1119-20.

18. Olson, S.: The problematic slit ventricle syndrome. A review of the literature and proposed algorithm for treatment. Pediatr Neurosurg. 2004; 40: 264-269.

19. Petrella, G., Czosnyka, M., Smielewski, P., Allin, D., Guazzo, E.P., Pickard, J.D., et al.: In vivo assessment of hydrocephalus shunt. Acta Neurol Scand. 2009; 120: $317-$ 323.

20. Poca, M., Sahuquillo, J.: Intracranial pressure monitoring and CSF dynamics in patients with neurological disorders: Indications and practical considerations. Neurologia. 2001; 16: 303-320.

21. Poca, M.A., Sahuquillo, J., Arribas, M., Baguena, M., Amoros, S., Rubio, E.: Fiberoptic intraparenchymal brain pressure monitoring with the camino V420 monitor: Reflections on our experience in 163 severely head-injured patients. J Neurotrauma. 2002; 19: 439-448.

22. Rekate, H.L.: Shunt-related headaches: The slit ventricle syndromes. Childs Nerv Syst. 2008; 24: 423-430.

23. Rekate, H.L.: The slit ventricle syndrome: Advances based on technology and understanding. Pediatr Neurosurg. 2004; 40: 259-263.

24. Rekate, H.L.: Classification of slit-ventricle syndromes using intracranial pressure monitoring. Pediatr Neurosurg. 1993; 19: 15-20.

25. Schuhmann, M.U., Sood, S., McAllister, J.P., Jaeger, M., Ham, S.D., Czosnyka, Z., et al.: Value of overnight monitoring of intracranial pressure in hydrocephalic children. Pediatr Neurosurg. 2008; 44: 269-279.

26. Tamaki, N., Kusunoki, T., Kose, S., Matsumoto, S.: Continuous intracranial pressure monitoring in normal pressure hydrocephalus--with special reference to clinical significance of B wave and prognostic criteria for CSF shunting. No To Shinkei. 1983; 35: 131-137.

Horcajadas, A.; Román, A.; Olivares, G.; Saura, E.; Jorques, A; Cordero, N.: Utilidad de la monitorizacion de la PIC en pacientes con sospecha de disfuncion valvular. Neurocirugía 2011; 22: 310-323.

Correspondencia: Cuesta del Pino 65 Casa 29. Granada 18008 